\title{
Antikonvulsiva und Schwangerschaft - was bedeutet das für die Mutter?
}

Fragestellung: Antikonvulsiva gelten bekanntermaßen als risikobehaftet, wenn es um das Thema erhöhte Missbildungsraten bei der Einnahme während der Schwangerschaft geht. Dies ist aber nur ein Aspekt. Was geschieht mit Müttern, die eine Epilepsie haben? Haben sie auch ein erhöhtes Risiko während und nach der Schwangerschaft? Wenn ja, wie sieht dies aus?

Hintergrund: 0,3 - 0,8 \% aller Frauen, die schwanger werden, leiden an einer Epilepsie. Die meisten Epilepsiepatientinnen müssen auch während der Schwangerschaft ihre antikonvulsive Therapie unverändert fortsetzen, um sich und das Ungeborene vor Anfällen und den daraus resultierenden Folgen zu schützen. Die damit verbundenen Probleme für das Ungeborene sind vielfach publiziert und gut bekannt. Dass die Mutter selber einem erhöhten Risiko ausgesetzt ist, hingegen weniger. So ist z.B. die mütterliche Mortalität bei Epilepsiepatientinnen um das zehnfache erhöht im Vergleich zu denjenigen ohne diese chronische Erkrankung. Auch kommt es überproportional häufiger zu Komplikationen in der Schwangerschaft und um den Geburtstermin herum, ein Problem, was meist nicht ausreichend fokussiert wird.

Viale L, Allotey J, Cheong-See F et al. for EBM CONNECT Collaboration. Epilepsy in pregnancy and reproductive outcomes: a systematic review and metaanalysis. Lancet $2015 \mathrm{http}: / / \mathrm{dx}$. doi.org/10.1016/SO1406736(15)00045-8
Patienten und Methodik: Die vorliegenden Daten beruhen auf einer ausführlichen Literaturrecherche mittels aller verfügbaren relevanten Suchmaschinen wie MEDLINE, Embase, Cochrane, AMED und CINAHL, unabhängig von der Sprache, in der publiziert wurde. Die Suchkriterien waren Schwangerschaften bei Epilepsiepatientinnen mit und ohne Medikamentenexposition (Antikonvulsiva) mit dem Ziel Komplikationen vor, während und nach der Geburt zu erfassen, einschließlich möglicher Probleme, die sich für das Neugeborene ergeben.

Ergebnisse: Von 7.050 für die Fragestellung relevanten Literaturstellen entsprachen 38 Studien den Auswertekriterien der Autoren, wobei in insgesamt 39 publizierten Artikeln 2.837.325 Schwangerschaften erfasst und ausgewertet worden waren. Die Daten stammten dabei sowohl aus Industrie- als auch aus Entwicklungsländern. Frauen mit Epilepsie hatten in der Zusammenschau aller Daten im Vergleich zu Frauen ohne Epilepsie ein erhöhtes Risiko für Spontanaborte, für erhöhte ante- und postpartale Blutungskomplikationen und für Blutdruckkrisen. Zudem fanden sich bei Epilepsiepatientinnen öfter Geburten mittels Kaiserschnitt oder Hinweise auf eine ärztlich eingeleitete Geburt. Die Kinder kamen häufiger vor dem geplanten Termin $(<37$ Gestationswoche) zur Welt und wiesen häufiger eine Wachstumsverzögerung auf, im Vergleich zur Normalbevölkerung. Kein Unterschied bestand in Bezug auf die Zahl der Totgeburten oder perinatalen Tod oder die Einweisung auf eine neonatale Intensivstation. Das Risiko, einen Schwangerschaftsdiabetes zu entwickeln, war in beiden Patientinnengruppen vergleichbar.

Schlussfolgerungen: Es existiert ein geringes, aber doch signifikant erhöhtes Risiko eines ungünstigeren Verlaufs der Schwangerschaft bei Frauen mit Epilepsie. Auffallend erhöht ist außerdem die Anzahl der Kaiserschnitte und der medikamentös eingeleiteten Geburten.

\section{- Kommentar von Vivien Homberg, Bad Berka}

\section{Mütter gefährdet}

Eine ausgesprochen gelungene Beantwortung einer viel zu wenig beachteten Fragestellung. Auch wenn solche Daten natürlich nicht die Validität und Qualität prospektiver Studien haben, ist es eine alltagsrelevante Fragestellung, definitiv nicht nur für Neurologen, sondern auch für Gynäkologen und Geburtshelfer. Das Risiko perinataler Komplikationen bei Epilepsiepatientinnen ist demnach erhöht, zwar nicht dramatisch, aber doch beachtenswert. Ein suffizientes Monitoring dieser Schwangerengruppe in der Perinatalperiode ist daher zwingend zu fordern, nicht nur was die Gründlichkeit der Ultraschalldiagnostik des Ungeborenen betrifft.

Mehr und validere Daten wären bei der vorliegenden Erkenntnis dringend von Nöten. Die Autoren selbst kritisieren, dass sie anhand der Daten nicht in der Lage waren, detaillierteren Fragen nachzugehen und zum Beispiel die Ursache der

Epilepsie, Anfallsschwere und -häufigkeit um den Geburtstermin herum, Medikamentenanamnese oder Dosis und verabreichte Antikonvulsiva mit den erhöhten Risiken zu korrelieren.

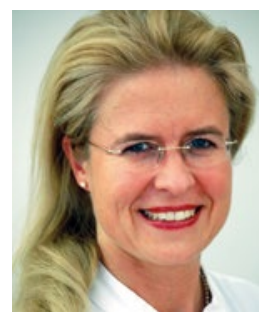

Dr. med. Vivien Homberg, Bad Berka

Chefärztin der Klinik für Neurologie, Zentralklinikum Bad Berka E-Mail:Vivien.Homberg@zentralklinik.de 